\title{
SÍNDROME DE BURNOUT, SATISFACCIÓN LABORAL E INTEGRACIÓN FAMILIAR EN TRABAJADORES DE UNA TIENDA POR DEPARTAMENTO DE AREQUIPA
}

\author{
BURNOUT SYNDROM, JOB SATISFACTION AND FAMILY \\ INTEGRATION IN WORKERS FROM A DEPARTMENT \\ STORE AT AREQUIPA
}

Walter L. Arias Gallegos y Karla D. Ceballos Canaza Universidad Católica San Pablo, Arequipa, Perú

\section{Resumen:}

El presente estudio, pretende analizar las relaciones entre tres variables: el síndrome de burnout, la satisfacción laboral y la integración familiar; dentro del marco de lo que se conoce como conflicto familia-trabajo. Para ello se ha tomado una muestra de 20 trabajadores de una tienda por departamentos, que está casada y tiene hijos. Los resultados señalan que los niveles de agotamiento, despersonalización y baja realización personal son severos en la mayoría de trabajadores. Su nivel de integración familiar es medio y existen diversas relaciones entre las variables. Un hallazgo relevante es que las dimensiones del síndrome de burnout explican la integración familiar en un sentido positivo y negativamente a la satisfacción laboral. Además la integración familiar podría tener un efecto amortiguador en la satisfacción laboral cuando el trabajador tiene niveles altos de estrés.

Palabras clave: Síndrome de burnout, satisfacción laboral, integración familiar, conflicto trabajo-familia. 


\section{Summary:}

The present study aims to analyze the relationships between three variables: syndrome of burnout, job satisfaction and family integration; within the framework of what is known as work-family conflict. For this we took a sample of 20 workers, who are married and have children. The results indicate that the levels of exhaustion, depersonalization and inefficacy are severe in the majority of workers. Their level of family integration is average and diverse relations exist between the variables. An important finding is that the dimensions of burnout syndrome explain the level of family integration in a positive sense and job satisfaction negatively. In addition, the family integration might have a dampening effect on job satisfaction when the workers has high levels of stress.

Key words: Burnout syndrome, job satisfaction, family integration, work-family conflict.

\section{Introducción}

El síndrome de burnout es una consecuencia del estrés laboral crónico que acarrea múltiples signos y síntomas, pero los más característicos a lo largo del tiempo que se ha venido investigando este fenómeno, son el agotamiento emocional, la despersonalización y la baja realización personal (Arias y Jiménez, 2012). Esta triada sindrómica obedece a las formulaciones teóricas de Maslach y Jackson (1981) que plantearon el síndrome de burnout como un factor de riesgo psicosocial producto del impacto del contacto prolongado que tienen los trabajadores de profesiones de servicios sociales con los destinatarios de su trabajo. De hecho, las profesiones más estudiadas han sido los profesores (de nivel inicial, primario, secundarios y superior) que tienen contacto con los alumnos, los trabajadores en el campo de la seguridad que responden a las necesidades de los ciudadanos (vale decir policías, fuerzas armadas, etc.) y el personal de salud (médicos, enfermeras, psicólogos, obstetrices, anestesiólogos, psiquiatras, etc.) que interactúa con las personas que tienen diversos padecimientos que afectan su salud física y/o mental.

En los últimos años, han cobrado mayor fuerza explicativa las teorías que se focalizan en los factores organizacionales, más que en las relaciones entre los trabajadores y los usuarios de su trabajo, además de que ya no se considera como una manifestación exclusiva de los grupos profesionales antes mencionados, sino que puede afectar a cualquier trabajador, siempre que existan riesgos psicosociales en su entorno laboral (Gil-Monte y Peiró, 1999).

En ese sentido, en Arequipa, se comenzó estudiando el síndrome de burnout en tres grupos profesionales de riesgo (enfermeras, profesores y policías) para después extender dichos estudios en otros grupos profesionales como administrativos, psicólogos, call-centers, supervisores de seguridad industrial; y valorar las relaciones entre éste síndrome y diversas variables laborales como el clima organizacional, y de corte clínico como los estilos de afrontamiento (Arias, 2015). 
En el presente estudio, vamos a centrarnos en dos variables más, una de corte organizacional como es la satisfacción laboral y otra de tipo familiar como es la integración familiar; considerando que en años recientes, se han hecho más patentes las necesidades de armonizar las demandas laborales con las actividades familiares (Guerrero y Puerto, 2007). En nuestra ciudad, un abordaje inicial, lo ha dado Belén Salvatierra (2011) que utilizó métodos de intervención familiar para concientizar a los trabajadores de diferentes centros mineros del Perú sobre la necesidad de asumir de manera más responsable las normas de seguridad en el trabajo, pues se había encontrado previamente que dadas las condiciones laborales de este grupo profesional, sus familias se sienten afectadas por el despilfarro y ciertas "malas costumbres" que adoptan al encontrase lejos de sus familias, y que derivan en problemas laborales.

Con respecto a la satisfacción laboral, existen diversos modelos teóricos que explican este constructo. Entre los principales factores podemos mencionar los ingresos económicos, las relaciones interpersonales con los compañeros de trabajo y los superiores, el reconocimiento, las posibilidades de ascenso y promoción en el trabajo, etc. (Baron, 1997). De hecho, existen varias teorías que explican la satisfacción laboral, entre las que se destacan la teoría de las necesidades de Maslow, la teoría tripartita de Alderfer, la teoría de la equidad de Adams, la teoría bifactorial de Herzberg, la teoría de las características del puesto de Hackman y Oldham, la teoría de las necesidades de logro de McClelland, etc. (véase Schultz, 1998).
En estudios previos, hemos podido constatar que la satisfacción laboral depende mucho del contexto organizacional (Arias y Justo, 2013) y que el clima organizacional se relaciona con la satisfacción laboral, sobre todo, las dimensiones de flexibilidad y de reconocimiento (Arias y Arias, 2014). Otros estudios locales han puesto énfasis en la felicidad y la satisfacción laboral (Gamero, 2013), pero son inexistentes, los estudios que relacionan la satisfacción laboral con la familia en Arequipa y el Perú.

Un abordaje a esta temática, la dimos cuando se diseñó y validó el Inventario de Integración Familiar, para lo cual se partió de definir la integración familiar como "el grado de salud, equilibrio y armonía de las relaciones que nacen del vínculo conyugal y que naturalmente se orientan a satisfacer la necesidad de trascendencia personal en base al respeto, el diálogo y la comunión entre sus miembros considerando sus responsabilidades, según el ciclo vital de la familia" (Arias, Castro, Dominguez, Masías, Canales, Castilla y Castilla, 2013, p. 196). En aquella ocasión se pudo evidenciar que los niveles de integración entre los pobladores de la ciudad de Arequipa eran bajos en un $62.6 \%$ de 844 pobladores de 13 distritos de Arequipa Metropolitana y que la integración familiar se relacionaba con el nivel socioeconómico, los ingresos económicos y el grado de instrucción (Castro, Arias, Dominguez, Masías, Salas, Canales y Flores, 2013).

Ahora bien, ¿cómo se relacionan, el síndrome de burnout, la satisfacción laboral y la integración familiar? Diversos estudios dan cuenta de que la satisfacción laboral viene mediada por factores laborales y por 
eventos familiares, a este tema de estudio se le conoce como el conflicto trabajo-familia o familia-trabajo. En ese sentido, considerando los cambios en la organización familiar que se han vivido en los últimos años, las relaciones entre la familia y el trabajo se han estrechado más, pues ahora, tanto el varón como la mujer, trabajan y tienen una vida familiar. Aunque todavía es la mujer quien asume más la carga familiar y el varón quien dedica más tiempo al trabajo, en ambos casos un espacio interfiere con el otro de manera cada vez más frecuente. En sentido, se ha encontrado que para los varones, la familia es fuente que perturba su quehacer en el trabajo, mientras que para las mujeres, el trabajo es fuente de insatisfacción en la familia (Quiroga y Sánchez, 1997).

Este conflicto puede ser fuente de estrés que de prolongarse en el tiempo podría manifestarse con la sintomatología que se presenta en el síndrome de burnout. Por ello, es importante que la familia sea fuente de apoyo para los trabajadores (Martínez-Pérez y Osca, 2002), pero también es muy útil que desde el trabajo se den políticas de apoyo para la familia, tema que en nuestro país se agota con las facilidades que se prestan para la maternidad y paternidad.

Una secuencia lógica sería que la integración familiar afecta la satisfacción laboral y si esta resulta afectada, aparezcan las manifestaciones del estrés. Por ejemplo, un estudio en 779 soldados, mostró que a mayor implicación con el trabajo, mayor es la satisfacción laboral, aunque el estrés sea elevado (López-Araújo, Osca y Peiró, 2007). Consideramos que la integración familiar parte de la satisfacción con la pareja que está mediada por la satisfacción sexual y la comunicación de la pareja (Eguiluz, Calvo y De la Orta, 2012), así como por la resolución adecuada de las crisis propias del desarrollo familiar, en función de su estructura, su ciclo vital y las dinámicas propias de sus miembros, que incluyen la asunción de roles familiares y la demarcación de límites claros entre sus integrantes (Arias, 2012).

Por otro lado, se ha demostrado que las demandas laborales son fuertes predictores del conflicto trabajo-familia, mientras que las demandas familiares predicen mejor el conflicto familia-trabajo (Sanz, 2011). En un estudio previo con enfermeras de tres hospitales de Arequipa, pudimos constatar que las trabajadoras que tenían mayor sobrecarga laboral y mayor número de hijos eran las que exhibían más síntomas de agotamiento emocional y del síndrome de burnout (Arias y Jiménez, 2011). Otros estudios han reportado que el conflicto trabajo-familia se relaciona con niveles elevados de agotamiento emocional, pero que ciertas variables personales como la autoeficacia, moderan los efectos del estrés causado por el conflicto trabajo-familia (Rubio, Osca, Recio, Urien y Peiró, 2015).

En el presente estudio, pretendemos analizar las relaciones entre el síndrome de burnout, la satisfacción familiar y la integración familiar en una muestra de trabajadores de una tienda por departamento de la ciudad de Arequipa. Además, se valorarán los efectos de cada una de las variables de estudio entre sí, a través del análisis de regresión lineal. 


\section{Metodología}

\section{Muestra}

La muestra está constituida por 20 trabajadores de una tienda por departamentos de la ciudad de Arequipa, considerando que solo se tomó como parte de la muestra a todos aquellos trabajadores que están casados y tienen hijos, y que aceptaron participar en el estudio de manera voluntaria. Las características de la muestra se detallan a continuación: $45 \%$ son varones $(\mathrm{N}=9)$ y $55 \%$ son mujeres $(\mathrm{N}=11)$. La edad promedio es de 34 años con una desviación estándar de \pm 8.2 dentro de un rango de 19 y 52 años de edad. La media del tiempo de relación es de 8.85 años, además, el 30\% tiene un hijo, otro $30 \%$ tiene dos, otro $30 \%$ más tiene tres hijos y solo el 10\% tiene cuatro hijos.

Con respecto al grado de instrucción, el $20 \%$ tiene estudios secundarios culminados, otro $20 \%$ tiene estudios técnicos y el $60 \%$ tiene estudios universitarios, ya sea que los haya culminado o no. Con respecto a su cargo, en la empresa, $5 \%$ son consultores, $20 \%$ son pre-vendedores, $55 \%$ son vendedores, $10 \%$ son cajeros y 1 trabajador ocupa el cargo de jefe ( $5 \%$ ) y solo uno (5\%), no lleno esta información.

En función de sus ingresos económicos, el $20 \%$ percibe un sueldo mensual de 900 soles, el $15 \%$ percibe un sueldo entre 950 y 1100 soles, $25 \%$ tiene un sueldo de 1150 soles, $15 \%$ percibe 1200 soles, el $20 \%$ gana entre 1400 y 1500 soles, $10 \%$ gana 1800 soles y $5 \%$ tiene ingresos mensuales de 1900 soles. El 25\% considera que se ubica en un nivel socioeconómico bajo y el 75\% en nivel socioeconómico medio.

\section{Instrumentos}

Ficha de registro sociolaboral que comprende datos tales como edad, sexo, grado de instrucción, la cantidad de ingresos, cargo, nivel socioeconómico, tiempo de relación con la pareja el y número de hijos.

Inventario de Burnout de Maslach (IBM), que fue validada Gil-Monte y Peiró (1999) en una muestra multiocupacional hispanohablante con un índice de confiabilidad de 0.79. Este inventario consta de 22 ítems de carácter autoafirmativo con una escala de frecuencia de 7 grados que va de o (nunca) a 6 (todos los días), tipo Likert. El instrumento se compone de tres subescalas que corresponden a las tres dimensiones del síndrome de burnout: agotamiento emocional (9 ítems), despersonalización (5 ítems) y baja realización personal (8 ítems). Se consideraron como puntos de corte de la escala total los siguientes valores: bajo, entre o y 44 puntos; moderado entre $45 \mathrm{y}$ 88; y severo, 89 a más. Estos valores han sido considerados en los estudios previos que ha realizado el autor principal (Arias y Jiménez, 2012; Arias, 2015).

Escala de Satisfacción en el Trabajo de Warr, Cook y Wall, que consta de 15 ítems y dos dimensiones: satisfacción laboral intrínseca y satisfacción laboral extrínseca. En una ocasión previa se han analizado las características psicométricas de este instrumento (Arias y Arias, 2014), que presenta correlaciones ítemtest por encima de 0.4 , con un estructura factorial obtenida mediante el método de componentes principales del análisis factorial exploratorio con rotación varimax, y un nivel de confiabilidad de $\alpha=0.763$, mediante 
el método de consistencia interna y la prueba Alfa de Cronbach.

El Inventario de Integración Familiar es un instrumento de medida unidimensional que consta de 52 ítems dispuestos en una estaca tipo likert que va de siempre (5) a nunca (1), que se aplica a personas jefes de familia varones o mujeres que tengan hijos. La prueba cuenta con criterios de validez de contenido, criterio y de constructo, además de que posee un índice de confiabilidad mediante la prueba Alfa de Cronbach de 0.739. Asimismo, cuenta con baremos en tres rangos de interpretación: bajo, moderado y alto (Arias, Castro, Dominguez, Masías, Canales, Castilla y Castilla, 2013).

\section{Procedimientos}

Las personas entrevistadas fueron evaluadas en su centro de trabajo, con el permiso de su jefe inmediato superior. Un colaborador fue quien recabó la información de los trabajadores, para lo cual fue debidamente entrenado sobre el uso de los instrumentos. Las aplicaciones de las pruebas se realizaron en un promedio de 30 minutos por persona, dentro de su horario de trabajo, durante los meses de julio y setiembre del año 2015.

\section{Resultados}

El puntaje medio del agotamiento emocional fue de 32.5 lo que implica que le ubica en los niveles de severo, la media de la despersonalización fue de 18.2, es decir muy severo, y la media de la baja realización personal fue de 31.15, es decir, también severo; mientras que el síndrome de burnout obtuvo una media de 82.3 vale decir, moderado alto. La satisfacción intrínseca tuvo una media de 28.25 , la satisfacción intrínseca fue de 33.65, ý la satisfacción laboral fue de 61.9; todos calores moderados. Finalmente la integración familiar obtuvo una media de 212.2, que se interpreta como moderada.

Tabla 1.

Valores descriptivos

\begin{tabular}{|c|c|c|c|c|c|c|c|c|}
\hline & 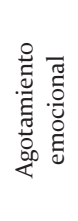 & 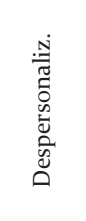 & 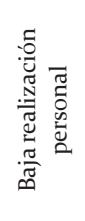 & 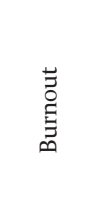 & 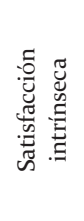 & 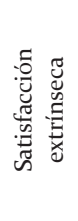 & 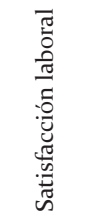 & 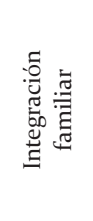 \\
\hline Media & 32.5 & 18.2 & 31.15 & 82.3 & 28.25 & 33.65 & 61.9 & 212.2 \\
\hline Mediana & 31 & 19 & 30 & 81.5 & $27 \cdot 5$ & 33 & $59 \cdot 5$ & 215 \\
\hline Moda & 30 & 12 & 20 & 96 & 24 & 33 & 51 & 226 \\
\hline Desv. típ. & 5.871 & 3.994 & 8.183 & 12.109 & 4.363 & 5.896 & $9 \cdot 480$ & 25.299 \\
\hline Varianza & 34.473 & 15.957 & 66.976 & 146.642 & 19.039 & 34.765 & 89.884 & 640.063 \\
\hline Asimetría & 0.394 & -0.367 & 0.451 & -0.227 & 0.762 & 0.885 & 0.866 & -0.365 \\
\hline Curtosis & 0.311 & -0.996 & 0.143 & -1.048 & -0.187 & 1.544 & -0.183 & -0.703 \\
\hline Mínimo & 21 & 12 & 18 & 58 & 22 & 23 & 51 & 157 \\
\hline Máximo & 45 & 24 & 50 & 100 & 38 & 49 & 82 & 247 \\
\hline
\end{tabular}


Asimismo, el 45\% tiene niveles bajos de integración familiar, 30\% tiene un nivel medio y $25 \%$ tiene un nivel alto. Con respecto al síndrome de burnout, $15 \%$ tiene niveles moderados de agotamiento emocional y $85 \%$ tiene niveles severos; el 100\% presenta niveles severos de despersonalización; el $15 \%$ tiene niveles leves de baja realización personal, $25 \%$ niveles moderados y $60 \%$ niveles severos. Finalmente, el 6o\% tiene niveles moderados del síndrome de burnout y 40\% niveles severos.

Tabla 2.

Correlaciones

\begin{tabular}{|c|c|c|c|c|c|c|c|c|c|c|c|c|}
\hline & Edad & $\begin{array}{c}\text { T. de } \\
\text { relación }\end{array}$ & $\begin{array}{c}\mathrm{N}^{\circ} \\
\text { hijos }\end{array}$ & Ingresos & $\begin{array}{c}\text { Integrac. } \\
\text { familiar }\end{array}$ & $\begin{array}{c}\text { Agot. } \\
\text { emocional }\end{array}$ & Desper. & $\begin{array}{c}\text { Baja } \\
\text { realización }\end{array}$ & Burnout & $\begin{array}{c}\text { Satisfac. } \\
\text { intrínseca }\end{array}$ & $\begin{array}{c}\text { Satisfac. } \\
\text { extrínseca }\end{array}$ & $\begin{array}{c}\text { Satisfac. } \\
\text { laboral }\end{array}$ \\
\hline 1 & 1 & $\mathbf{0 . 7 9 1}$ & $\mathbf{0 . 4 1 9}$ & 0.068 & $\mathbf{0 . 2 8 0}$ & $\mathbf{- 0 . 4 1 0}$ & 0.102 & $\mathbf{- 0 . 4 3 7}$ & $\mathbf{- 0 . 5 0 5}$ & -0.075 & 0.048 & -0.004 \\
\hline 2 & & 1 & $\mathbf{0 . 6 4 0}$ & 0.194 & $\mathbf{0 . 4 1 9}$ & $\mathbf{- 0 . 2 2 8}$ & 0.196 & $\mathbf{- 0 . 3 9 2}$ & $\mathbf{- 0 . 3 6 2}$ & $\mathbf{- 0 . 3 1 3}$ & -0.156 & $\mathbf{- 0 . 2 4 1}$ \\
\hline 3 & & & 1 & 0.150 & $\mathbf{0 . 5 7 9}$ & -0.087 & 0.115 & $\mathbf{- 0 . 2 5 0}$ & $\mathbf{- 0 . 2 2 0}$ & $\mathbf{- 0 . 2 4 6}$ & -0.043 & -0.140 \\
\hline 4 & & & & 1 & -0.119 & $\mathbf{0 . 2 3 3}$ & $\mathbf{0 . 4 6 9}$ & 0.143 & $\mathbf{0 . 3 8 1}$ & -0.127 & -0.025 & -0.074 \\
\hline 5 & & & & & 1 & $\mathbf{- 0 . 3 6 3}$ & -0.086 & -0.075 & $\mathbf{- 0 . 3 4 7}$ & 0.029 & $\mathbf{0 . 2 2 8}$ & 0.155 \\
\hline 6 & & & & & & 1 & $\mathbf{0 . 2 1 3}$ & $\mathbf{0 . 3 6 3}$ & $\mathbf{0 . 7 7 6}$ & $\mathbf{- 0 . 2 4 9}$ & $\mathbf{- 0 . 4 5 3}$ & $\mathbf{- 0 . 3 9 7}$ \\
\hline 7 & & & & & & & 1 & $\mathbf{- 0 . 2 8 7}$ & $\mathbf{0 . 2 4 2}$ & $\mathbf{- 0 . 4 2 8}$ & $\mathbf{- 0 . 4 0 1}$ & $\mathbf{- 0 . 4 4 6}$ \\
\hline 8 & & & & & & & & 1 & $\mathbf{0 . 7 6 2}$ & 0.166 & $\mathbf{0 . 2 9 4}$ & $\mathbf{0 . 2 6 0}$ \\
\hline 9 & & & & & & & & & 1 & -0.068 & -0.075 & -0.078 \\
\hline 10 & & & & & & & & & & 1 & $\mathbf{0 . 7 0 1}$ & $\mathbf{0 . 8 9 6}$ \\
\hline 11 & & & & & & & & & & & 1 & $\mathbf{0 . 9 4 4}$ \\
\hline 12 & & & & & & & & & & & & 1 \\
\hline
\end{tabular}

Se realizaron también análisis de correlación mediante la prueba de Pearson (Tabla 1), encontrando que la edad se relaciona con el tiempo de relación y el número de hijos de manera positiva (alta, moderada y baja, respectivamente, $\mathrm{r}=0.791, \mathrm{r}=0.419 \mathrm{y} \mathrm{r}=0.280$ ), pero negativamente con el agotamiento emocional $(\mathrm{r}=-0.40)$, la baja realización personal $(\mathrm{r}=$ -o.437) y el puntaje total del Inventario de Burnout de Maslach ( $\mathrm{r}=-0.505)$. El tiempo de relación se relacionó con el número de hijos de manera moderada alta $(r=0.640)$, con la integración familiar de manera moderada ( $\mathrm{r}=$ o.419). Además, mantuvo correlaciones bajas y negativas con el agotamiento emocional $(\mathrm{r}=-\mathbf{0 . 2 2 8})$, la baja realización personal $(\mathrm{r}=$ -0.392), el síndrome de burnout ( $\mathrm{r}=-0.362)$, la satisfacción laboral intrínseca $(\mathrm{r}=-0.313)$ y la satisfacción laboral ( $\mathrm{r}=-0.241)$. El número de hijos se relacionó moderada y positivamente con la integración familiar $(\mathrm{r}=0.579)$ y negativamente con la baja realización personal $(\mathrm{r}=$ -0.250), el síndrome de burnout $(\mathrm{r}=-0.220) \mathrm{y}$ la satisfacción laboral intrínseca $(\mathrm{r}=-0.246)$, pero estas correlaciones fueron bajas. Los ingresos mensuales se relacionaron baja pero positivamente con el agotamiento emocional $(r=0.233)$, la despersonalización $(r=0.469) y$ el síndrome de burnout ( $\mathrm{r}=0.381)$.

La integración familiar se relacionó moderada y negativamente con el agotamiento emocional ( $\mathrm{r}=-0.363)$ y el síndrome de burnout $(\mathrm{r}=-0.347)$, pero baja y positivamente con la satisfacción laboral extrínseca $(\mathrm{r}=\mathbf{0 . 2 2 8})$. El agotamiento emocional se relacionó con la despersonalización, la baja realización personal, el síndrome de burnout, siendo ésta última correlación, alta 
$(\mathrm{r}=\mathrm{0} .776)$, mientras que se relacionó negativamente pero de manera baja y moderada con la satisfacción laboral y sus dos factores. La despersonalización se relacionó de manera baja y negativa con la baja realización personal, y de manera baja y positiva con el síndrome de burnout. Además, se relacionó de manera negativa y moderada con la satisfacción laboral y sus dos factores ( $r>-0.4)$. La baja realización personal se relacionó de manera alta y positiva con el síndrome de burnout $(\mathrm{r}=0.762)$ y de manera baja y positiva con la satisfacción laboral y su factor de satisfacción extrínseca. La satisfacción laboral intrínseca se relacionó positiva y fuertemente con la satisfacción laboral ( $\mathrm{r}=$ $0.896)$ y su factor extrínseco ( $\mathrm{r}=0.701)$, al igual que este factor, se relacionó con la satisfacción laboral ( $\mathrm{r}=0.944)$ (ver Tabla 1).

Tabla 3.

Comparaciones según el sexo

\begin{tabular}{|c|c|c|c|c|c|c|c|}
\hline & Sexo & $\mathrm{N}$ & Media & $\begin{array}{c}\text { Desviación } \\
\text { típica }\end{array}$ & $\mathrm{t}$ & gl & $\mathrm{p}$ \\
\hline \multirow{2}{*}{ Tiempo de relación } & Varón & 9 & 6.333 & 6.726 & \multirow{2}{*}{-1.327} & \multirow{2}{*}{18} & \multirow{2}{*}{0.200} \\
\hline & Mujer & 11 & 10.909 & 8.677 & & & \\
\hline \multirow{2}{*}{$\mathrm{N}^{\circ}$ de hijos } & Varón & 9 & 1.777 & 1.201 & \multirow{2}{*}{-0.842} & \multirow{2}{*}{18} & \multirow{2}{*}{0.413} \\
\hline & Mujer & 11 & 2.181 & 0.873 & & & \\
\hline \multirow{2}{*}{ Ingresos mensuales } & Varón & 9 & 1244.444 & 282.228 & \multirow{2}{*}{0.287} & \multirow{2}{*}{18} & \multirow{2}{*}{0.777} \\
\hline & Mujer & 11 & 1204.545 & 339.451 & & & \\
\hline \multirow{2}{*}{ Agotamiento emocional } & Varón & 9 & 32.555 & 5.659 & \multirow{2}{*}{0.037} & \multirow{2}{*}{18} & \multirow{2}{*}{0.970} \\
\hline & Mujer & 11 & 32.454 & 6.314 & & & \\
\hline \multirow{2}{*}{ Despersonalización } & Varón & 9 & 15.555 & 3.972 & \multirow{2}{*}{-3.155} & \multirow{2}{*}{18} & \multirow{2}{*}{0.007} \\
\hline & Mujer & 11 & 20.363 & 2.500 & & & \\
\hline \multirow{2}{*}{ Baja realización personal } & Varón & 9 & 34.777 & 9.162 & \multirow{2}{*}{1.841} & \multirow{2}{*}{18} & \multirow{2}{*}{0.087} \\
\hline & Mujer & 11 & 28.181 & 6.209 & & & \\
\hline \multirow{2}{*}{ Síndrome de burnout } & Varón & 9 & 84 & 13.285 & \multirow{2}{*}{0.549} & \multirow{2}{*}{18} & \multirow{2}{*}{0.590} \\
\hline & Mujer & 11 & 80.909 & 11.519 & & & \\
\hline \multirow{2}{*}{ Satisfacción laboral intrínseca } & Varón & 9 & 30.333 & 5.361 & \multirow{2}{*}{1.956} & \multirow{2}{*}{18} & \multirow{2}{*}{0.050} \\
\hline & Mujer & 11 & 26.545 & 2.464 & & & \\
\hline \multirow{2}{*}{ Satisfacción laboral extrínseca } & Varón & 9 & 35.666 & 7.382 & \multirow{2}{*}{1.339} & \multirow{2}{*}{18} & 0205 \\
\hline & Mujer & 11 & 32 & 3.974 & & & 0.205 \\
\hline Soticf ió 1 hbor & Varón & 9 & 66 & 11.895 & 1725 & 18 & 0111 \\
\hline Satislaccion Iadoral & Mujer & 11 & 58.545 & 5.483 & $1 . / 30$ & 10 & 0.111 \\
\hline
\end{tabular}

Como parte de nuestro procesamiento, valoramos comparativamentelosvalores obtenidos de las variables de estudio según ciertos criterios sociolaborales, para ello se aplicó la prueba t de student y el análisis de varianza. En función del sexo, solo se registraron diferencias significativas en despersonalización, siendo las mujeres las que tienen puntajes más altos, y en satisfacción laboral intrínseca, siendo los varones quienes tienen puntajes más elevados. Al hacer comparaciones con respecto al grado deinstrucción, mediante el análisis de varianza no se encontraron diferencias significativas en ninguna de las variables de estudio, tampoco se encontraron diferencias significativas al hacer comparaciones en función del cargo. 
Tabla 4 .

Comparaciones según el nivel socioeconómico

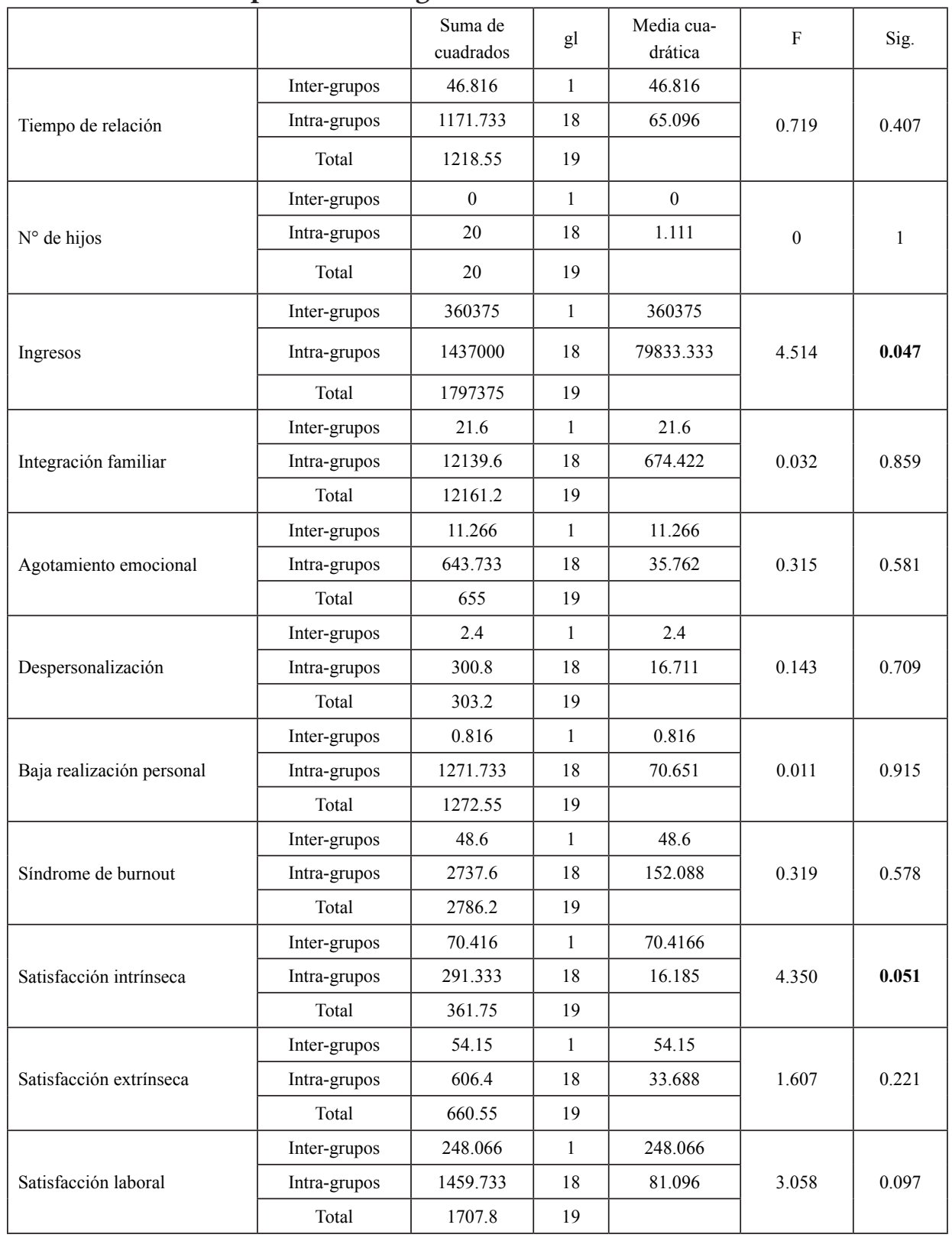


Sin embargo, sí se registraron diferencias en función del nivel socioeconómico. En la Tabla 4 se aprecian las comparaciones efectuadas mediante el análisis de varianza, donde se tiene que los ingresos económicos y la satisfacción laboral intrínseca presentan diferencias significativas según el nivel socioecoómico de los trabajadores.

Tabla 5 .

Coeficientes de regresión del Modelo 1

\begin{tabular}{|c|c|c|c|c|c|c|c|c|c|c|}
\hline \multirow{2}{*}{ Modelo 1} & \multicolumn{2}{|c|}{$\begin{array}{l}\text { Coeficientes no } \\
\text { estandarizados }\end{array}$} & \multirow{2}{*}{$\begin{array}{c}\begin{array}{c}\text { Coeficientes } \\
\text { estandarizados }\end{array} \\
\text { Beta }\end{array}$} & \multirow{2}{*}{$\mathrm{t}$} & \multirow{2}{*}{ Sig. } & \multicolumn{3}{|c|}{ Correlaciones } & \multicolumn{2}{|c|}{$\begin{array}{c}\text { Estadísticos } \\
\text { de colinealidad }\end{array}$} \\
\hline & B & Error típ. & & & & $\begin{array}{l}\text { Orden } \\
\text { cero }\end{array}$ & Parcial & Semiparcial & Tolerancia & FIV \\
\hline (Constante) & 110.977 & 35.753 & & 3.103 & 0.009 & & & & & \\
\hline Edad & -0.757 & 0.565 & -0.512 & -1.339 & 0.205 & -0.505 & -0.360 & -0.284 & 0.307 & 3.250 \\
\hline $\begin{array}{l}\text { Tiempo de } \\
\text { relación }\end{array}$ & 0.035 & 0.713 & 0.023 & 0.050 & 0.960 & -0.362 & 0.014 & 0.010 & 0.202 & 4.938 \\
\hline $\mathrm{N}^{\circ}$ de hijos & 0.156 & 3.763 & 0.013 & 0.041 & 0.967 & -0.220 & 0.011 & 0.008 & 0.443 & 2.252 \\
\hline Ingresos & 0.014 & 0.009 & 0.376 & 1.630 & 0.128 & 0.381 & 0.425 & 0.346 & 0.844 & 1.184 \\
\hline $\begin{array}{l}\text { Satisfacción } \\
\text { intrínseca }\end{array}$ & -0.259 & 0.884 & -0.093 & -0.293 & 0.773 & -0.068 & -0.084 & -0.062 & 0.444 & 2.248 \\
\hline $\begin{array}{l}\text { Satisfacción } \\
\text { extrínseca }\end{array}$ & 0.149 & 0.660 & 0.072 & 0.226 & 0.824 & -0.075 & 0.065 & 0.048 & 0.436 & 2.291 \\
\hline $\begin{array}{l}\text { Integración } \\
\text { familiar }\end{array}$ & -0.091 & 0.140 & -0.190 & -0.647 & 0.529 & -0.347 & -0.183 & -0.137 & 0.523 & 1.910 \\
\hline
\end{tabular}

Finalmente, un procesamiento que también se realizó, fue el análisis de regresión con la finalidad de predecir los efectos de cada una de las variables según diversos modelos de predicción. En un primer modelo se tomó al síndrome de burnout como variable dependiente y a edad, la los ingresos económicos, el número de hijos, el tiempo de relación de los trabajadores con sus respectivas parejas, la integración familiar, la satisfacción laboral y sus dos factores (intrínseco y extrínseco) como variables independientes o predictoras. El modelo planteado no resultó significativo $(\mathrm{p}<0.272)$ y ninguna de las variables tomadas como independientes tuvieron el valor predictivo sobre la aparición del síndrome de burnout (ver Tabla 5). 
Tabla 6.

Coeficientes de regresión del Modelo 2

\begin{tabular}{|c|c|c|c|c|c|c|c|c|c|c|}
\hline \multirow{2}{*}{ Modelo 2} & \multicolumn{2}{|c|}{$\begin{array}{l}\text { Coeficientes no } \\
\text { estandarizados }\end{array}$} & \multirow{2}{*}{$\begin{array}{c}\begin{array}{c}\text { Coeficientes } \\
\text { estandarizados }\end{array} \\
\text { Beta }\end{array}$} & \multirow{2}{*}{$\mathrm{t}$} & \multirow{2}{*}{ Sig. } & \multicolumn{3}{|c|}{ Correlaciones } & \multicolumn{2}{|c|}{$\begin{array}{c}\text { Estadísticos } \\
\text { de colinealidad }\end{array}$} \\
\hline & B & Error típ. & & & & $\begin{array}{l}\text { Orden } \\
\text { cero }\end{array}$ & Parcial & Semiparcial & Tolerancia & FIV \\
\hline (Constante) & -17.617 & 29.487 & & -0.597 & 0.563 & & & & & \\
\hline Edad & 0.795 & 0.299 & 0.688 & 2.658 & 0.023 & -0.004 & 0.643 & 0.358 & 0.270 & 3.698 \\
\hline Tiempo de relación & -0.793 & 0.329 & -0.670 & -2.408 & 0.036 & -0.241 & -0.605 & -0.324 & 0.233 & 4.277 \\
\hline $\mathrm{N}^{\circ}$ de hijos & -1.992 & 1.990 & -0.215 & -1.000 & 0.340 & -0.140 & -0.301 & -0.134 & 0.390 & 2.560 \\
\hline Ingresos & 0.006 & 0.005 & 0.223 & 1.283 & 0.228 & -0.074 & 0.376 & 0.172 & 0.597 & 1.674 \\
\hline $\begin{array}{l}\text { Agotamiento } \\
\text { emocional }\end{array}$ & -3.092 & 0.664 & -1.914 & -4.655 & 0.000 & -0.397 & -0.827 & -0.626 & 0.107 & 9.331 \\
\hline Despersonalización & -4.213 & 0.925 & -1.775 & -4.551 & 0.001 & -0.446 & -0.821 & -0.612 & 0.119 & 8.393 \\
\hline $\begin{array}{l}\text { Baja realiz. } \\
\text { personal }\end{array}$ & -3.141 & 0.840 & -2.711 & -3.736 & 0.003 & 0.260 & -0.763 & -0.503 & 0.034 & 29.051 \\
\hline $\begin{array}{l}\text { Síndrome de } \\
\text { burnout }\end{array}$ & 3.251 & 0.781 & 4.152 & 4.162 & 0.001 & -0.078 & 0.796 & 0.560 & 0.018 & 54.902 \\
\hline $\begin{array}{l}\text { Integración } \\
\text { familiar }\end{array}$ & 0.294 & 0.091 & 0.784 & 3.227 & 0.009 & 0.155 & 0.714 & 0.434 & 0.306 & 3.261 \\
\hline
\end{tabular}

En un segundo modelo se tomó a la satis- $\quad$ planteado resulto significativo $(\mathrm{p}<\mathrm{0.009})$ facción laboral como variable dependientey y los coeficientes beta estandarizados de a la edad, el tiempo de relación, el número la edad, el síndrome de burnout y la intede hijos, los ingresos económicos, el agota- gración familiar predicen positivamente la miento emocional, la despersonalización, satisfacción laboral, mientras que el tiempo la baja realización personal, el síndrome de relación, el agotamiento emocional, la de burnout y la integración familiar como despersonalización y la baja realización variables independientes. El resultado, personal predicen negativamente la satisse aprecia en la Tabla 6, donde el modelo facción laboral. 
Tabla 7.

Coeficientes de regresión del Modelo 3

\begin{tabular}{|c|c|c|c|c|c|c|c|c|c|c|}
\hline \multirow{2}{*}{ Modelo 3} & \multicolumn{2}{|c|}{$\begin{array}{c}\text { Coeficientes } \\
\text { no estandarizados }\end{array}$} & \multirow{2}{*}{$\begin{array}{c}\begin{array}{c}\text { Coeficientes } \\
\text { estandarizados }\end{array} \\
\text { Beta }\end{array}$} & \multirow{2}{*}{$\mathrm{t}$} & \multirow{2}{*}{ Sig. } & \multicolumn{3}{|c|}{ Correlaciones } & \multicolumn{2}{|c|}{$\begin{array}{c}\text { Estadísticos } \\
\text { de colinealidad }\end{array}$} \\
\hline & B & Error típ. & & & & $\begin{array}{l}\text { Orden } \\
\text { cero }\end{array}$ & Parcial & Semiparcial & Tolerancia & FIV \\
\hline (Constante) & 163.234 & 54.299 & & 3.006 & 0.014 & & & & & \\
\hline Edad & -1.859 & 0.787 & -0.602 & -2.360 & 0.042 & 0.280 & -0.618 & -0.304 & 0.255 & 3.907 \\
\hline $\begin{array}{l}\text { Tiempo de } \\
\text { relación }\end{array}$ & 1.710 & 0.894 & 0.541 & 1.911 & 0.088 & 0.419 & 0.537 & 0.246 & 0.207 & 4.808 \\
\hline $\mathrm{N}^{\circ}$ de hijos & 9.236 & 4.507 & 0.374 & 2.049 & 0.070 & 0.579 & 0.564 & 0.264 & 0.499 & 2.002 \\
\hline Ingresos & -0.017 & 0.013 & -0.211 & -1.273 & 0.234 & -0.119 & -0.390 & -0.164 & 0.604 & 1.655 \\
\hline $\begin{array}{l}\text { Agotamiento } \\
\text { emocional }\end{array}$ & 6.749 & 2.090 & 1.566 & 3.228 & 0.010 & -0.363 & 0.732 & 0.416 & 0.070 & 14.113 \\
\hline $\begin{array}{l}\text { Despersonali- } \\
\text { zación }\end{array}$ & 10.151 & 2.408 & 1.602 & 4.215 & 0.002 & -0.086 & 0.814 & 0.544 & 0.115 & 8.669 \\
\hline $\begin{array}{l}\text { Baja realiz. } \\
\text { personal }\end{array}$ & 8.335 & 1.839 & 2.696 & 4.530 & 0.001 & -0.075 & 0.833 & 0.585 & 0.047 & 21.232 \\
\hline $\begin{array}{l}\text { Síndrome de } \\
\text { burnout }\end{array}$ & -8.155 & 1.888 & -3.903 & -4.318 & 0.001 & -0.347 & -0.821 & -0.557 & 0.020 & 48.982 \\
\hline $\begin{array}{l}\text { Satisfacción } \\
\text { intrínseca }\end{array}$ & 1.672 & 1.218 & 0.288 & 1.373 & 0.202 & 0.029 & 0.416 & 0.177 & 0.377 & 2.646 \\
\hline $\begin{array}{l}\text { Satisfacción } \\
\text { extrínseca }\end{array}$ & 1.789 & 1.109 & 0.417 & 1.612 & 0.141 & 0.228 & 0.473 & 0.208 & 0.249 & 4.011 \\
\hline
\end{tabular}

En un tercer modelo, se asumió como variable dependiente a la integración familiar, y como variables predictoras a la edad, el tiempo de relación, el número de hijos, los ingresos económicos, el agotamiento emocional, la despersonalización, la baja realización personal, el síndrome de burnout, la satisfacción laboral, el factor intrínseco y el factor extrínseco de la satisfacción laboral. Los resultados del análisis de regresión, señalan que este tercer modelo también es significativo ( $\mathrm{p}<0.011$ ), y que el agotamiento emocional, la despersonalización, y la baja realización personal predicen de manera positiva la integración familiar; en tanto que la edad y el síndrome de burnout predicen la integración familiar de manera negativa.

\section{Discusión}

En el presente estudio evaluamos las relaciones entre tres variables: el síndrome de burnout, la satisfacción laboral y la integración familiar; además de recoger información sociolaboral en 20 trabajadores casados y con hijos que se desempeñan haciendo diversas funciones en una tienda por departamentos de la ciudad. Al respecto, este grupo ocupacional, presenta condiciones laborales muy particulares, según la empresa para la que trabaje, y sus niveles de satisfacción laboral pueden verse afectados por diversos factores como los ingresos, sus relaciones con otros trabajadores, la estructura de la organización, sus posibilidades de ascenso, etc.; como pudimos constatarlo en un estudio previo 
(Arias y Justo, 2013). En esta ocasión hemos analizado las relaciones entre las variables de estudio mencionadas, con el fin de valorar el valor predictivo que tienen ellas entre sí.

Se puede apreciar en primer lugar que, la edad, el tiempo de relación y número de hijos se relacionan fuertemente entre sí, como era de esperarse, pues a medida que se incrementa la edad, es más probable tener más hijos y tener relaciones perdurables en el tiempo. La edad también predijo positivamente la satisfacción laboral y negativamente la integración familiar. La integración familiar también se relacionó con la edad, debido quizás al nivel de madurez emocional que tienen las personas con el paso del tiempo, y que se ha relacionado con el establecimiento de matrimonios más sólidos (Murillo, 2015), pero dados los resultados del análisis de regresión, el tener más edad predice una menor integración familiar. Posiblemente, esto se debe a que con el paso del tiempo, los hijos desde que son adolescentes se van distanciando un poco más de la familia, hasta que se independizan y forman la suya propia, como parte del ciclo vital natural (Arias, 2012). En todo caso, hace falta indagar más este tema para poder obtener resultados concluyentes.

Precisamente, la integración familiar se relacionó moderada y negativamente con el agotamiento emocional y el síndrome de burnout, que implica que quienes tienen familias más integradas, tienen menos niveles de estrés. La integración familiar también se relacionó de manera positiva pero baja con la satisfacción laboral extrínseca, lo que puede fundamentarse en hallazgos previos donde se encontró que las personas con familias más integradas, perciben mayores ingresos económicos, se ubican en un nivel socioeconómico más alto y poseen un grado de instrucción más elevado (Castro et al, 2013). Sin embargo, a nivel predictivo, el síndrome de burnout explica positivamente la satisfacción laboral, mientras que las tres dimensiones del síndrome explican la satisfacción laboral negativamente. Estas incongruencias, pueden deberse a las críticas hechas por estudios psicométricos en los que se analiza el uso inadecuado de los puntos de corte del síndrome de burnout (Fernández y Merino, 2014). Consideramos que tendría más peso el hallazgo obtenido mediante la regresión, vale decir que el agotamiento emocional, la despersonalización y la baja realización personal predicen mayor insatisfacción laboral en los trabajadores, ya que la satisfacción laboral se relacionó negativamente con el agotamiento emocional y la despersonalización de manera moderada, lo que quiere decir que a mayor agotamiento y despersonalización menor será la satisfacción laboral. Aquí es importante destacar que los valores en estas dimensiones fueron elevados, ya que $85 \%$ tiene niveles severos de agotamiento emocional, $100 \%$ presenta niveles severos de despersonalización y $60 \%$ tiene niveles severos de baja realización personal.

Por otra parte, el síndrome de burnout predijo negativamente la integración familiar, pero sus tres dimensiones predicen negativamente la integración familiar. Esto significa que a mayor burnout menor es la integración familiar y que a mayor agotamiento, despersonalización y baja realización personal, mayor será la integración 
familiar. Una explicación a esta incongruencia, radica nuevamente en los puntos de corte de la escala total del inventario de Maslach; pero los pesos predictivos de las dimensiones del burnout, podrían sugerir, que cuando el trabajador tiene se siente más agotado, distante y desmotivado, su familia se integra más, brindándole apoyo. Sin embargo, para poder asegurar esto, es necesario evaluar otras variables y valorar sus relaciones y causas. Esto empero, es plausible porque la integración familiar tiene un efecto positivo en la satisfacción laboral según los coeficientes beta obtenidos mediante el análisis de regresión.

Para terminar, la satisfacción laboral no tuvo peso predictivo en la integración familiar ni en el síndrome de burnout, lo que quiere decir que las dimensiones del síndrome de burnout tienen un efecto mediador entre la satisfacción familiar y la integración familiar. De manera global, estos datos sugieren que el agotamiento emocional, la despersonalización y la baja realización personal explican la negativamente la satisfacción laboral y positivamente la integración familiar. Por tanto, una secuencia lógica de las tres variables en su conjunto es que las dimensiones del síndrome de burnout generan más integración familiar, que a su vez deviene en mayores niveles de satisfacción laboral, mientras que la satisfacción laboral disminuye en la medida que aumenta el agotamiento emocional, la despersonalización y la baja realización personal. Por ende, la integración familiar estaría cumpliendo un rol amortiguador del estrés laboral en la satisfacción del trabajador (ver Figura 1).

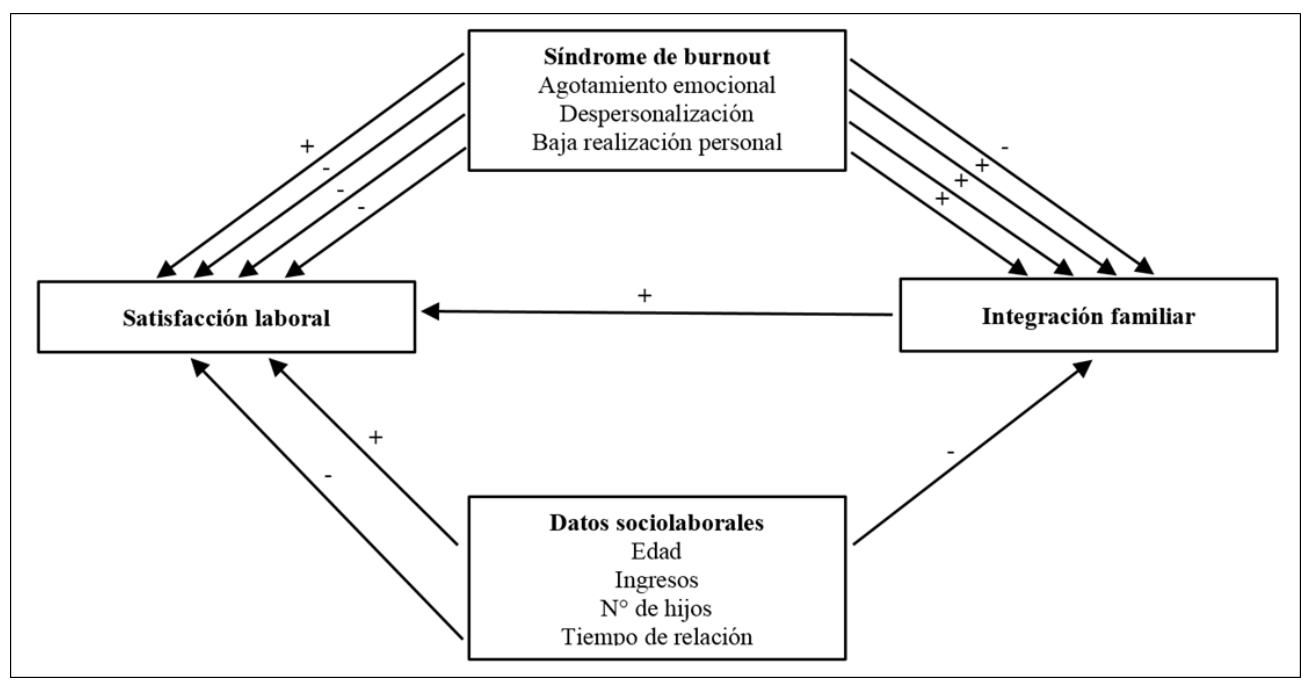

Figura 1.

Gráfico del comportamiento predictivo de las variables 
Estos resultados empero, no son concluyentes por varias razones. Primero tiene que ver el tamaño de la muestra, que por considerar solo a 20 personas, no puede generalizar los hallazgos a otros trabajadores de otras empresas. En segundo lugar, es necesario contar con instrumentos estandarizados, pues los niveles de validez y confiabilidad han sido obtenidos en otros grupos ocupacionales. En tercer lugar, se deben valorar otras variables como el apoyo social o familiar, los estilos de afrontamiento y diversas características del trabajo y la empresa. Como cuarta limitación, podríamos señalar que un procesamiento más adecuado que nos permitiría confirmar nuestros hallazgos sería el análisis factorial confirmatorio o el método de las ecuaciones estructurales.

A pesar de estas deficiencias, nuestro estudio tiene importancia notable, pues a pesar de las contradicciones expuestas, parece perfilarse el rol que juega cada una de estas variables en el conflicto trabajo-familia. A ello se suma que este es el primer estudio realizado en nuestra ciudad que se avoca a esta temática tan compleja pero tan resonante en los últimos años. Es necesario en ese sentido, que la investigación de las variables familiares, se integre al estudio de los fenómenos laborales y organizacionales.

\section{Referencias}

Arias, W. L. (2012). Algunas consideraciones sobre la familia y la crianza desde un enfoque sistémico. "Revista de Psicología de Arequipa”, 2(1), 32-46.

Arias, W. L. (2015). “Síndrome de burnout y afrontamiento”. Arequipa: Adrus.
Arias, W. L. y Arias, G. (2014). Relación entre el clima organizacional y la satisfacción laboral en una pequeña empresa del sector privado. "Ciencia \& Trabajo", 16(51), 185-191.

Arias, W. L. y Jiménez, N. A. (2011). Incidencia del síndrome de burnout en enfermeras de los hospitales de Arequipa. “Nuevos Paradigmas", 5(1), 37-50.

Arias, W. L. y Jiménez, N. A. (2012). “Estrés crónico en el trabajo: Estudioso sobre el síndrome de burnout en Arequipa”. Arequipa: Universidad Católica San Pablo.

Arias, W. L. y Justo, O. (2013). Satisfacción laboral en trabajadores de dos tiendas por departamento: un estudio comparativo. "Ciencia \& Trabajo", 15(47), 41-46.

Arias, W. L.; Castro, R.; Dominguez, S.; Masías, M. A.; Canales, F.; Castilla, S. y Castilla, S. (2013). Construcción de un inventario de integración familiar. "Avances en Psicología”, 21(2), 195-206.

Baron, R. (1997). "Psicología”. México: Prentice Hall.

Castro, R.; Arias, W. L.; Dominguez, S.; Masías, M. A.; Salas, X.; Canales, F. \& Flores, A. (2013). Integración familiar y variables socioeconómicas en Arequipa metropolitana. "Revista de Investigación”, 4, 35-65.

Fernández, M. y Merino, C. (2014). Error de medición alrededor de los puntos 
de corte en el MBI-GS. "Liberabit", 20(2), 209-218.

Gamero, H. Y. (2013). La satisfacción laboral como dimensión de la felicidad. "Ciencia \& Trabajo”, 15(47), 94-102.

Gil-Monte, P. R. \& Peiró, J. M. (1999). Perspectivas teóricas y modelos interpretativos para el estudio del síndrome de quemarse por el trabajo. "Anales de Psicología”, 15(2), 261-268.

Guerrero, J. y Puerto, Y. I. (2007). Productividad, trabajo y salud: La perspectiva psicosocial. "Revista Colombiana de Psicología”, 16, 203-234.

Eguiluz, L. L.; Calvo, R. M. y De la Orta, D. (2012). Relación entre la percepción de la satisfacción marital, sexual y la comunicación en parejas. "Revista Peruana de Psicología y Trabajo Social", 1(1), 15-28.

López-Araújo, B.; Osca, A. y Peiró, J. M. (2007). El papel modulador de la implicación con el trabajo en la relación entre el estrés y la satisfacción laboral. "Psicothema", 19(1), 81-87.

Martínez-Pérez, M. D. y Osca, A. (2002). Análisis psicométrico del Inventario de Apoyo Familiar para Trabajadores. "Psicothema", 14(2), 310-316.

Maslach, C. \& Jackson, S. E. (1981). The measurement of experienced burnout. "Journal of Occupational Behavior", 12, 99-113.

Murillo, M. G. (2015). Desarrollo del juicio moral en personas casadas y divorciadas de la ciudad de Arequipa. Avances en Psicología, 23(1), 73-85.

Rubio, C.; Osca, A.; Recio, P.; Urien, B. y Peiró, J. M. (2015). Work-family conflict, self-efficacy, and emotional exhaustion: A test of longitudinal effects. "Journal of Work and Organizational Psychology", 31, 147-154.

Salvatierra, B. (2011). "Cultura de seguridad laboral para el minero”. Lima: Noceda.

Sanz, A. I. (2011). Conciliación y salud laboral: ¿Una relación posible? Actualidad en el estudio del conflicto trabajo-familia y la recuperación del estrés. "Medicina y Seguridad del Trabajo", 57(1), 115-126.

Schultz, D. (1998). "Psicología industrial”. México: McGraw-Hill.

Recibido: 04-02-2016 Aceptado: 09-05-2016 